\title{
Mapping photonic entanglement into and out of a quantum memory
}

\author{
K. S. Choi * H. Deng * J. Laurat ${ }^{\dagger}$, and H. J. Kimbl田 \\ Norman Bridge Laboratory of Physics 12-33, California Institute of Technology, Pasadena, California 91125, USA
}

(Dated: February 2, 2008)

\begin{abstract}
Recent developments of quantum information science [1] critically rely on entanglement, an intriguing aspect of quantum mechanics where parts of a composite system can exhibit correlations stronger than any classical counterpart [2]. In particular, scalable quantum networks require capabilities to create, store, and distribute entanglement among distant matter nodes via photonic channels [3]. Atomic ensembles can play the role of such nodes [4]. So far, in the photon counting regime, heralded entanglement between atomic ensembles has been successfully demonstrated via probabilistic protocols [5, 6]. However, an inherent drawback of this approach is the compromise between the amount of entanglement and its preparation probability, leading intrinsically to low count rate for high entanglement. Here we report a protocol where entanglement between two atomic ensembles is created by coherent mapping of an entangled state of light. By splitting a single-photon [7, 8, 9] and subsequent state transfer, we separate the generation of entanglement and its storage [10]. After a programmable delay, the stored entanglement is mapped back into photonic modes with overall efficiency of $17 \%$. Improvements of single-photon sources [11] together with our protocol will enable "on-demand" entanglement of atomic ensembles, a powerful resource for quantum networking.
\end{abstract}

In the quest to achieve quantum networks over long distances [3], an area of considerable activity has been the interaction of light with atomic ensembles comprised of a large collection of identical atoms [4, 12, 13]. In the regime of continuous variables, a particularly notable advance has been the teleportation of quantum states between light and matter [14]. For discrete variables with photons taken one by one, important achievements include the efficient mapping of collective atomic excitations to single photons $15,16,17,18,19]$, the realization of entanglement between a pair of distant ensembles [5, 20] and, more recently, entanglement distribution involving two pairs of ensembles [6]. The first step toward

\footnotetext{
†Present Address : Laboratoire Kastler Brossel, Université Paris 6, Ecole Normale Superieure et CNRS, UPMC Case 74, 4 place Jussieu, 75252 Paris Cedex 05, France
}

entanglement swapping has been made [21] and lightmatter teleportation has been demonstrated [22].

In all these cases, progress has relied upon probabilistic schemes following the measurement-induced approach developed in the seminal paper by Duan, Lukin, Cirac and Zoller [4] $(D L C Z)$ and subsequent extensions. For the $D L C Z$ protocol, heralded entanglement is generated by detecting a single photon emitted indistinguishably by one of two ensembles. Intrinsically, the probability $p$ to prepare entanglement with only 1 excitation shared between two ensembles is related to the quality of entanglement, since the likelihood for contamination of the entangled state by processes involving 2 excitations scales as $p$ [20], and results in low success probability for each trial. Although the degree of stored entanglement can approach unity for the (rare) successful trials [20], the condition $p \ll 1$ dictates reductions in count rate and compromises in the quality of the resulting entangled state (e.g., as $p \rightarrow 0$, processes such as stray light scattering and detector dark counts become increasingly important). Furthermore, for finite memory time, subsequent connection of entanglement becomes increasingly challenging [21].

The separation of processes for the generation of entanglement and for its storage enables this drawback to be overcome. Here, we demonstrate such a division by way of reversible mapping of an entangled state into a quantum memory. The mapping is obtained by using adiabatic passage based upon dynamic Electromagnetically Induced Transparency (EIT) [23, 24, 25, 26] (See Appendix). Storage and retrieval of a single photon have been demonstrated previously [27, 28]. Adiabatic transfer of a collective excitation has been demonstrated between two ensembles coupled by a cavity mode [29], which can provide a suitable approach for generating ondemand entanglement over short distances. However, for efficient distribution of entanglement over quantum networks, reversible mapping of an entangled state between matter and light, as illustrated in Fig. 1h, has not been addressed until now.

In our experiment, entanglement between two atomic ensembles $L_{a}, R_{a}$ is created by first splitting a single photon into two modes $L_{i n}, R_{i n}$ to generate an entangled state of light [7, 8, [9]. This entangled field state is then coherently mapped to an entangled matter state for $L_{a}, R_{a}$. On demand, the stored atomic entanglement for $L_{a}, R_{a}$ is reversibly converted back into entangled photonic modes $L_{\text {out }}, R_{\text {out }}$. As opposed to the original $D L C Z$ scheme, our approach is inherently deterministic, suffering principally from the finite efficiency with 


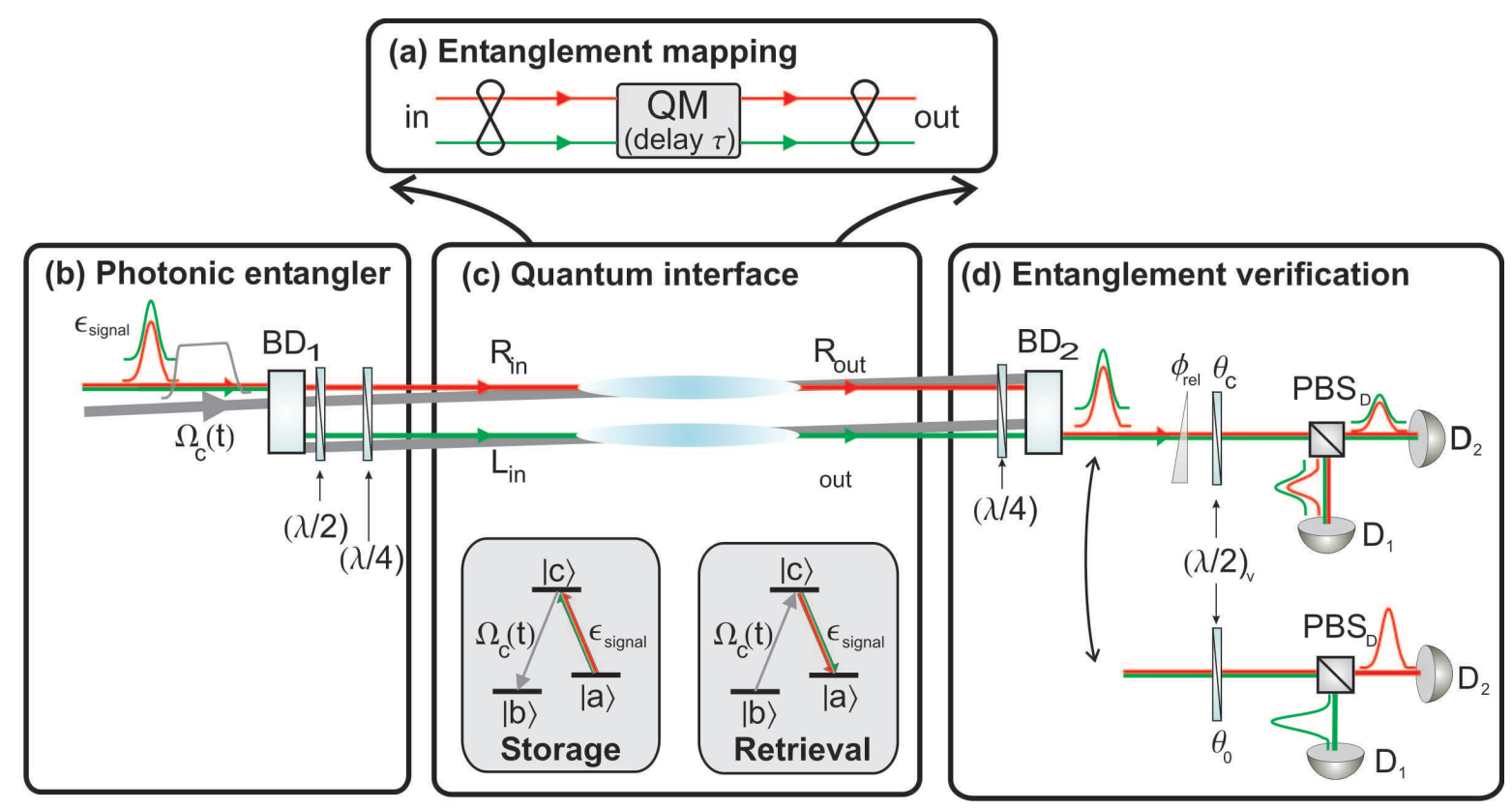

FIG. 1: Overview of the experiment. a, Illustration of the mapping of an entangled state of light into and out of a quantum memory (QM) with storage time $\tau$. b, Photonic "entangler": A beam displacer $B D_{1}$ splits an input single photon into two orthogonally polarized, entangled modes $L_{i n}, R_{i n}$, which are spatially separated by $1 \mathrm{~mm}$. With waveplates $\lambda / 2$ and $\lambda / 4$, the signal fields $\epsilon_{\text {signal }}$ for $L_{i n}, R_{i n}$ and control fields $\Omega_{c}^{(L, R)}(t)$ are transformed to circular polarizations with the same helicity along each path $L, R$, and copropagate with angle of $3^{\circ}$. c, Reversible mapping : Photonic entanglement between $L_{i n}, R_{i n}$ is coherently mapped into the memory ensembles $L_{a}, R_{a}$ by switching $\Omega_{c}^{(L, R)}(t)$ off adiabatically. After a programmable storage time, the atomic entanglement is reversibly mapped back into optical modes $L_{\text {out }}, R_{\text {out }}$ by switching $\Omega_{c}^{(L, R)}(t)$ on. Relevant energy diagrams for the storage and retrieval processes are shown in the insets. States $|a\rangle$, $|b\rangle$ are the hyperfine ground states $F=4, F=3$ of $6 S_{1 / 2}$ in atomic cesium; state $|c\rangle$ is the hyperfine level $F^{\prime}=4$ of the electronic excited state $6 P_{3 / 2}$. d, Entanglement verification : After a $\lambda / 4$ plate, the beam displacer $B D_{2}$ combines modes $L_{\text {out }}, R_{\text {out }}$ into one beam with orthogonal polarizations. With $(\lambda / 2)_{v}$ at $\theta_{c}=22.5^{\circ}$ before the polarization beamsplitter $\left(\mathrm{PBS}_{D}\right)$, single photon interference is recorded at detectors $D_{1}, D_{2}$ by varying the relative phase $\phi_{\text {rel }}$ by a Berek compensator. With $(\lambda / 2)_{v}$ at $\theta_{0}=0^{\circ}$, photon statistics for each mode $L_{\text {out }}, R_{\text {out }}$ are measured independently.

which single excitations can be mapped to and from an atomic memory, which can approach $45 \%$ [30]. Moreover, the contamination of entanglement for the $L_{a}, R_{a}$ ensembles from processes involving 2 excitations can be arbitrarily suppressed (independent of the mapping probabilities) with continuing advances in on-demand single photon sources 11]. Our experiment thereby provides a promising avenue to distribute and store entanglement deterministically over remote atomic ensembles for quantum networks [10].

The experimental setup is depicted in Fig. 1. Our single photon source is based on Raman transitions in an optically thick cesium ensemble [4, 15] (See Appendix). This system generates 25 ns-long single photons (resonant with the $6 S_{1 / 2}, F=4 \leftrightarrow 6 P_{3 / 2}, F^{\prime}=4$ transition) in a heralded fashion [15]. The single photons are polarized at $45^{\circ}$ from the eigen-polarizations of the beam displacer $B D_{1}$ (Fig. 1b), which splits them into entangled optical modes $L_{i n}, R_{i n}$ (called the signal modes) to produce, in the ideal case, the state $\frac{1}{\sqrt{2}}\left(\left|0_{L_{i n}}\right\rangle\left|1_{R_{i n}}\right\rangle+e^{i \phi_{r e l}}\left|1_{L_{i n}}\right\rangle\left|0_{R_{i n}}\right\rangle\right)$.
The next stage consists in coherently mapping photonic entanglement for $L_{i n}, R_{i n}$ into atomic ensembles $L_{a}, R_{a}$ (called the memory ensembles) within a single cloud of cold cesium atoms in a magneto-optical trap (MOT) (Fig. 1c). Ensembles $L_{a}, R_{a}$ are defined by the well-separated optical paths of the entangled photonic modes $L_{i n}, R_{i n}$. To avoid dissipative absorption for the fields in modes $L_{i n}, R_{i n}$ for our choice of polarization [28], we initially spin-polarize the atomic ensemble into $\left|F=4, m_{F}=0\right\rangle$ (See Appendix). A synchronous clock governs the trials of both the single photon source and memory ensembles with a period of 575 ns. Initially, the strong control fields $\Omega_{c}^{(L, R)}$ (resonant with the $6 S_{1 / 2}, F=3 \leftrightarrow 6 P_{3 / 2}, F^{\prime}=4$ transition) open transparency windows $\Omega_{c}^{(L, R)}(0)$ in $L_{a}, R_{a}$ for the signal modes. As the wavepacket of the signal field propagates through each ensemble, the control fields $\Omega_{c}^{(L, R)}(t)$ are turned off in 20 ns by an intensity modulator, thus coherently transforming the fields of the respective signal modes to collective atomic excitations within $L_{a}, R_{a}$. This mapping leads to heralded entanglement between 
ensembles $L_{a}, R_{a}$. After a user-defined delay, chosen here to be $1.1 \mu \mathrm{s}$, the atomic entanglement is converted back into entangled photonic modes by switching on the control fields $\Omega_{c}^{(L, R)}(t)$ (See Appendix).

For a given optical depth $\gamma$, there is an optimal Rabi frequency $\Omega_{c}(t)$ for the control field. In our experiment, the measured $\gamma$ and $\Omega_{c}(0)$ are 15 and $24 \mathrm{MHz}$, respectively. An example of our measurements of the EIT process for a single ensemble is presented in Fig. 2, which shows in (a) the input single-photon pulse and in (b) its storage and retrieval (See Appendix). Due to finite $\gamma$ and small length $(\approx 3 \mathrm{~mm})$ of the ensemble, there is considerable loss in the storage process, as evidenced by the counts around $\tau=0 \mu \mathrm{s}$ in (b). The peak beyond $\tau=1$ $\mu \mathrm{s}$ represents the retrieved pulse after $1.1 \mu \mathrm{s}$ of storage. Overall, we find good agreement between our measurements and the results from a numerical calculation following the methods of [26], using the fitted function of the input signal field (Fig. 2a) as the initial condition with all other parameters from independent measurements. The overall storage and retrieval efficiency is measured to be $\eta_{r}=17 \pm 1 \%$, also in agreement with the simulation.

With these results in hand for the individual $L_{a}, R_{a}$ ensembles, we next turn to the question of verification of entanglement for the input $L_{i n}, R_{\text {in }}$ and output $L_{\text {out }}, R_{\text {out }}$ optical modes. We follow the protocol introduced in Ref. [5] by (1) reconstructing a reduced density matrix $\rho$ constrained to a subspace containing no more than one excitation in each mode, and (2) assuming that all offdiagonal elements between states with different numbers of photons vanish, thereby obtaining a lower bound for any purported entanglement. In the photon-number basis $\left|n_{L}, m_{R}\right\rangle$ with $\{n, m\}=\{0,1\}$, the reduced density matrix $\rho$ is written as [5]

$$
\rho=\frac{1}{P}\left(\begin{array}{cccc}
p_{00} & 0 & 0 & 0 \\
0 & p_{01} & d & 0 \\
0 & d^{*} & p_{10} & 0 \\
0 & 0 & 0 & p_{11}
\end{array}\right) .
$$

Here, $p_{i j}$ is the probability to find $i$ photons in mode $L_{k}$ and $j$ in mode $R_{k}, d \simeq \frac{V\left(p_{01}+p_{10}\right)}{2}$ is the coherence between $\left|1_{L} 0_{R}\right\rangle_{k}$ and $\left|0_{L} 1_{R}\right\rangle_{k}, P=p_{00}+p_{01}+p_{10}+p_{11}$, and $V$ is the visibility for interference between modes $L_{k}, R_{k}$, with $k \in\{$ in out $\}$. The degree of entanglement of $\rho$ can be quantified in terms of the concurrence $C=$ $\frac{1}{P} \max \left(0,2|d|-2 \sqrt{p_{00} p_{11}}\right)$ which is a monotone function of entanglement, ranging from 0 for a separable state to 1 for a maximally entangled state 31 .

Operationally, the various elements of $\rho$ are obtained by recombining the $L_{k}, R_{k}$ fields with a second beam displacer, $B D_{2}$, as illustrated in Fig. 1 1 , to obtain a single spatial mode with orthogonal polarizations for the $L_{k}, R_{k}$ fields $[\underline{6}, 20]$. The diagonal elements of $\rho$ are measured with $(\lambda / 2)_{v}$ set at $0^{\circ}$ so that detection events at $D_{1}, D_{2}$ are recorded directly for the $L_{k}, R_{k}$ fields. To
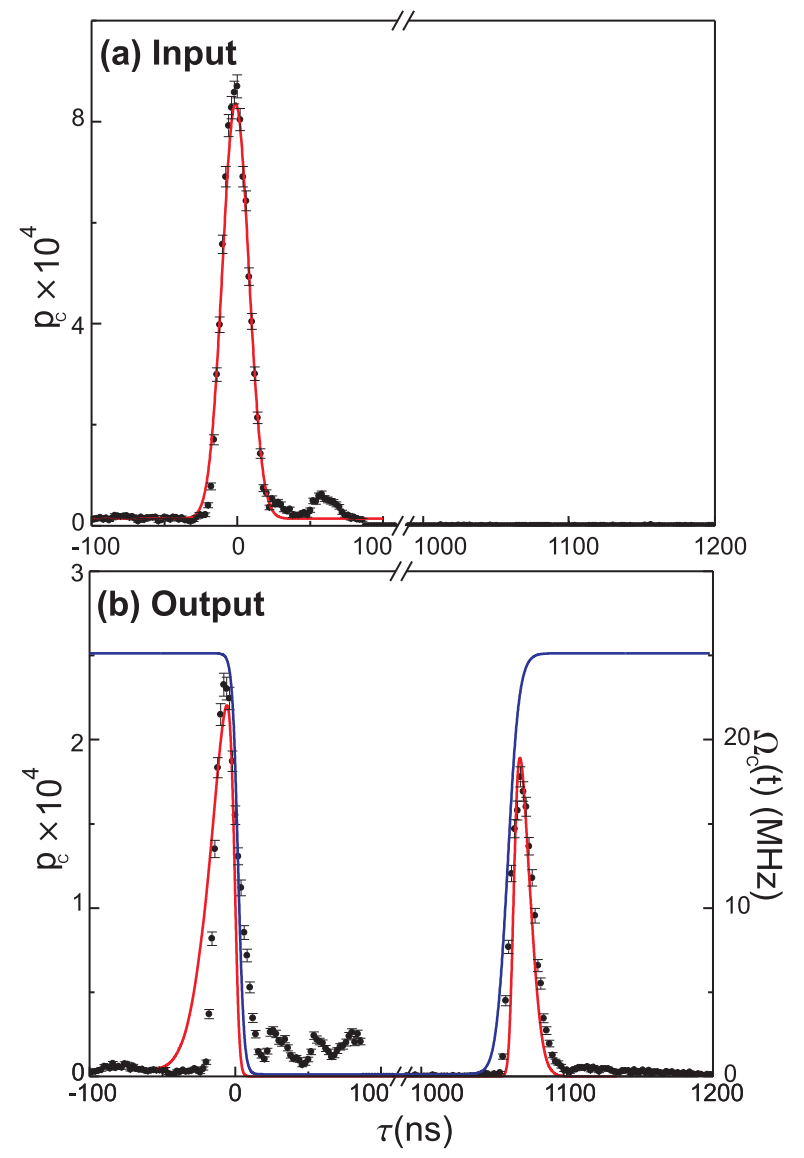

FIG. 2: Single photon storage and retrieval for a single ensemble. a, Input: The measured probability density $p_{c}$ for signal field, here a single photon generated from a separate atomic ensemble [15]. The red solid line represents a gaussian fit of $1 / e$ width of $28 \mathrm{~ns}$. $\mathbf{b}$, Storage and retrieval : The points around $\tau=0 \mu$ s represent "leakage" of the signal field due to the finite optical depth and length of the ensemble. The points beyond $\tau=1 \mu$ s show the retrieved signal field. The overall storage and retrieval efficiency is $17 \pm 1 \%$. The blue solid line is the estimated Rabi frequency $\Omega_{c}(t)$ of the control pulse. The red solid curve is from a numerical calculation solving the equation of motion of the signal field in a dressed medium 26]. Error bars give the statistical error for each point.

determine the off-diagonal components of $\rho$, the modes $L_{k}, R_{k}$ are brought into interference with $(\lambda / 2)_{v}$ set at $22.5^{\circ}$, as shown in Fig. 1 $\mathrm{d}$. By varying the relative phase $\phi_{\text {rel }}$ between the modes, we determine the visibility for single-photon interference and thereby deduce $d$.

We first perform tomography on the input modes $L_{i n}, R_{i n}$ to verify that they are indeed entangled. To this end, we remove the memory ensembles to transmit directly the signal fields into the verification stage, following our protocol of complementary measurements. The interference fringe between the two input modes is shown in Fig. 3a. From the independently determined propagation and detection efficiencies, our measurements 


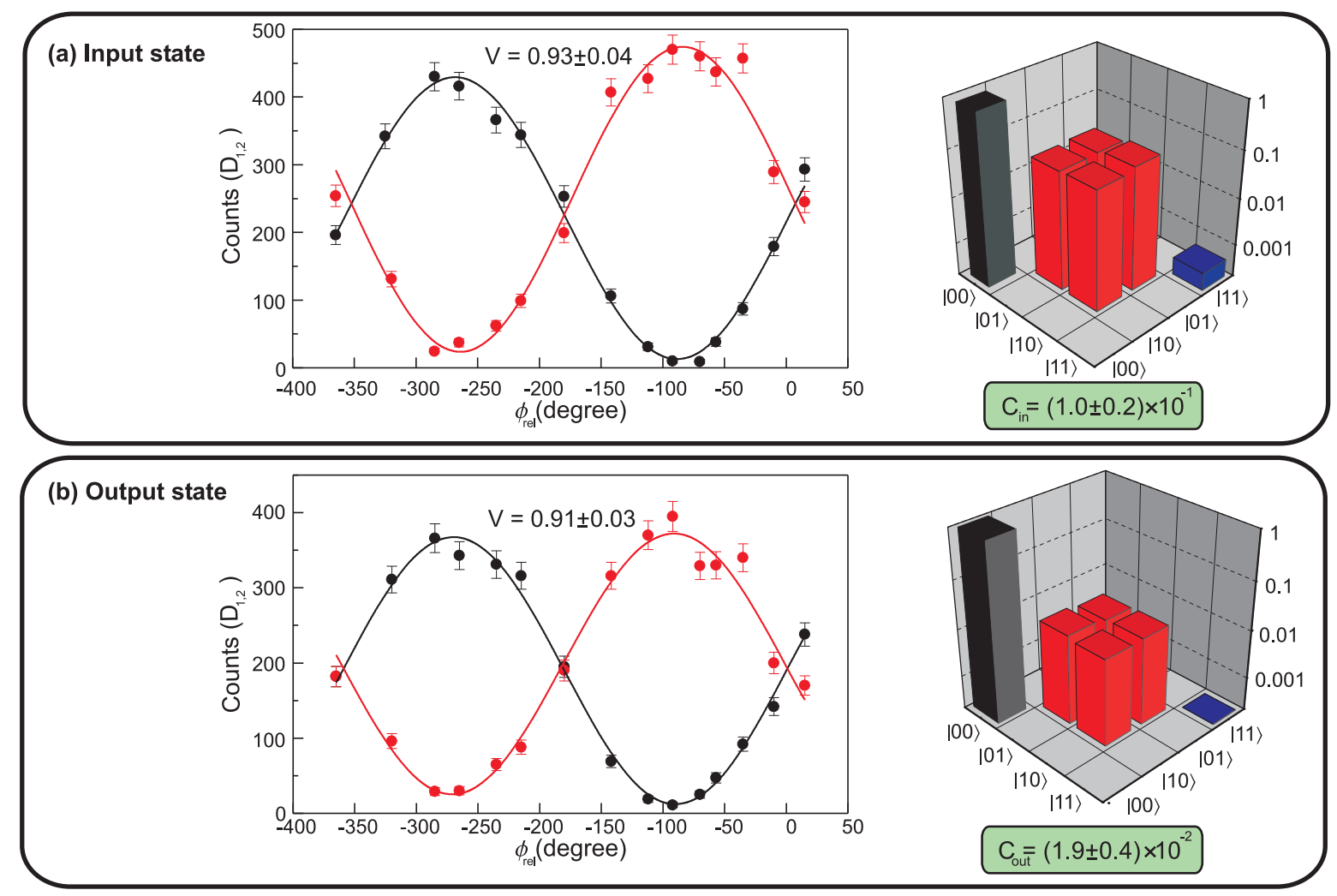

FIG. 3: Entanglement for the input (a) and output (b) optical modes. To verify entanglement, complementary measurements are performed : interference leading to a fringe when the relative phase $\phi_{\text {rel }}$ is scanned and photon statistics for the light modes separately. The figure shows the interference fringes and the reconstructed density matrices (in log scale) for the photonic modes $\mathbf{a}$, at the input of the memory and $\mathbf{b}$, at the output after storage and retrieval. The estimated concurrence is given in each case. Each point of the fringe is taken for 20,000 (100,000) heralding events for the input (output) state. Error bars indicate statistical errors.

at $D_{1}, D_{2}$ can be used to infer the quantum state for the input modes $L_{i n}, R_{i n}$ entering the input faces of the atomic ensembles $L_{a}, R_{a}$ [5], with the reconstructed density matrix $\rho_{i n}$ also given in Fig. 3k. The concurrence derived from $\rho_{\text {in }}$ is $C_{\text {in }}=0.10 \pm 0.02$, so that the fields for $L_{i n}, R_{i n}$ are indeed entangled. The value of the concurrence is in good agreement with the independently derived expectation of $C_{i n}^{\text {theory }}=0.10 \pm 0.01$, which depends on the quality of the single photon and the vacuum component (i.e., the overall efficiency) 20]. Given a heralding click from our single photon source, the probability to have a single photon at the face of either memory ensemble is $15 \%$, leading to a vacuum component of $85 \%$. We also independently characterize the suppression $w$ of the two-photon component relative to a coherent state (for which $w=1$ ) and find $w=0.09 \pm 0.03$. Our input entanglement is only limited by the current properties of our single-photon source, which will be improved with the rapid advances in sources of single photons [11].

Having verified entanglement for the input modes $L_{i n}, R_{i n}$, we next map this photonic entanglement into the $L_{a}, R_{a}$ ensembles, which serve as a quantum memory (Fig. 15). After storing the entanglement for $1.1 \mu \mathrm{s}$, we transfer the resulting atomic excitation from the memory to the output modes $L_{\text {out }}, R_{\text {out }}$ and perform quantumstate tomography to determine $\rho_{\text {out }}$ precisely as for $\rho_{\text {in }}$. As shown in Fig. 3, the visibility for interference of the fields after storage and retrieval shows no appreciable degradation (from $V_{\text {in }}=0.93 \pm 0.04$ to $V_{\text {out }}=0.91 \pm 0.03$ ). From the measurements at $D_{1}, D_{2}$, we infer the quantum state $\rho_{\text {out }}$ at the output faces of the $L_{a}, R_{a}$ ensembles, with the result displayed in Fig. 3 $\mathrm{b}$. The associated concurrence $C_{\text {out }}=(1.9 \pm 0.4) \times 10^{-2}$ is in agreement with $C_{\text {out }}^{\text {theory }}=(1.7 \pm 0.1) \times 10^{-2}$. Since the mapping of the atomic states from $L_{a}, R_{a}$ into field modes $L_{\text {out }}, R_{\text {out }}$ is a local operation, this measurement provides a lower bound for the entanglement between the $L_{a}, R_{a}$ ensembles [5]. Thus, we demonstrate the reversible mapping of an entangled state of the electromagnetic field to and from a material system. For completeness, Table I gives the diagonal elements and concurrences of $\bar{\rho}_{\text {in }}, \bar{\rho}_{\text {out }}$ determined directly at $D_{1}, D_{2}$ without correction for propagation and detection efficiencies.

We emphasize that although the entanglement associated with $\rho_{\text {out }}$ is heralded (because of the nature of our source of single photons), our protocol for generation 
and storage of entanglement is intrinsically deterministic. The transfer efficiency of entanglement from input modes to output modes of the quantum memory is limited by the storage and retrieval efficiency $\eta_{r}$ of the EIT process. This transfer can be quantified by the ratio $\lambda$ of the concurrence $C_{\text {out }}$ for the output state $\rho_{\text {out }}$ to $C_{\text {in }}$ for the input state $\rho_{i n}$. For an ideal source of single photons on-demand (with no vacuum component), the input concurrence is approximated by $C_{\text {in }} \simeq \alpha V$, where $\alpha$ denotes the transmission efficiency of the single photon from the source to the entangler in Fig. 10 [20]. Similarly, for the output, $C_{\text {out }} \simeq \alpha \eta_{r} V$, where we assume that the visibility $V$ is preserved by the mapping processes. Thus, $\lambda=\frac{C_{\text {out }}}{C_{\text {in }}} \simeq \eta_{r}$, which therefore estimates the maximum amount of entanglement in modes $L_{\text {out }}, R_{\text {out }}$ for the case of an (ideal) single photon generated deterministically. In our experiment, the entanglement transfer reaches $\lambda=(20 \pm 5) \%$.

The performance of our quantum interface depends also on the memory lifetime $\tau_{m}$ over which one can faithfully retrieve a stored quantum state. For our system, independent measurements of $\eta_{r}$ made by varying the storage duration $\tau$ allow us to determine $\tau_{m}=8 \pm 1 \mu \mathrm{s}$, as limited by inhomogeneous Zeeman broadening [32] and misalignment between the quantization axis and the bias magnetic field (See Appendix). Active and passive compensations of the residual magnetic field would improve $\tau_{m}$ [33], along with improved optical trapping techniques.

In conclusion, our work provides the first realization of mapping an entangled state into and out of a quantum memory. Our protocol alleviates the significant drawback of probabilistic protocols [4], where low preparation probabilities prevent its potential scalability [21], and thus our strategy leads to efficient scaling for high-fidelity quantum communication [10]. Our current results are limited by the large vacuum component of our available single photon source, which principally reduces the degree of entanglement in the input, and by the limited retrieval efficiency of the EIT process, which bounds the entanglement transfer $\lambda=20 \pm 5 \%$. However, with extension to an on-demand single photon source and with improved retrieval efficiency, pushed already to $45 \%$ in Ref. 30] by larger optical depth and optimum pulse shaping,

TABLE I: Experimentally determined diagonal elements $\bar{p}_{i j}$ and concurrences $\bar{C}_{\text {in }}, \bar{C}_{\text {out }}$ for the density matrices $\bar{\rho}_{\text {in }}, \bar{\rho}_{\text {out }}$ derived directly from detectors $D_{1}, D_{2}$ without correction for losses and detection efficiencies. The error bars indicate statistical errors.

\begin{tabular}{|lcc|}
\hline & $\bar{\rho}_{\text {in }}$ & $\bar{\rho}_{\text {out }}$ \\
\hline $\bar{p}_{00}$ & $0.9800 \pm 0.0001$ & $0.99625 \pm 0.00003$ \\
$\bar{p}_{10}$ & $(1.043 \pm 0.008) \times 10^{-2}$ & $(2.09 \pm 0.02) \times 10^{-3}$ \\
$\bar{p}_{01}$ & $(0.957 \pm 0.008) \times 10^{-2}$ & $(1.67 \pm 0.02) \times 10^{-3}$ \\
$\bar{p}_{11}$ & $(8 \pm 2) \times 10^{-6}$ & $(2 \pm 2) \times 10^{-7}$ \\
$\bar{C}$ & $(1.28 \pm 0.09) \times 10^{-2}$ & $(2.5 \pm 0.5) \times 10^{-3}$ \\
\hline
\end{tabular}

our protocol provides an alternate strategy for generating and distributing entanglement between remote quantum memories that circumvents some difficulties with the original $D L C Z$ protocol [4].

\section{APPENDIX}

\section{Experimental details.}

A $22 \mathrm{~ms}$ preparation stage and $3 \mathrm{~ms}$ experiment run are conducted every $25 \mathrm{~ms}$ period. During the preparation stage, atomic ensembles are loaded in a MOT for $18 \mathrm{~ms}$ and further cooled by optical molasses for $3 \mathrm{~ms}$. For $800 \mu \mathrm{s}$, we optically pump the atomic ensembles to the $6 S_{1 / 2}, F=4, m_{F}=0$ state in atomic cesium. During this stage, the trapping beam is turned off while the intensity of the repumping beam is reduced to $0.1 I_{\text {sat }}$. The quantization axis is chosen along the $k$-vector of the signal modes and defined by a pulsed magnetic field of 0.2 G. A pair of counter-propagating Zeeman pumping beams (10 MHz red-detuned from $4 \leftrightarrow 4^{\prime}$ and linearly polarized along the quantization axis) illuminate the ensembles in a direction perpendicular to modes $L_{i n}, R_{i n}$. The MOT repumping beam serves as a hyperfine pumping beam. The experiment is conducted at repetition rate of $1.7 \mathrm{MHz}$ during a $3 \mathrm{~ms}$ interval before the next MOT loading cycle. A small bias field of $10 \mathrm{mG}$ is left on to define the quantization axis for the experiment.

\section{Single photon generation.}

The single-photon source is based upon the protocol [4, 15] composed of time-delayed photon pairs, called fields 1,2 emitted from a cesium ensemble in a MOT called the source ensemble, located $3 \mathrm{~m}$ from the memory ensembles. For photon-pair production, a sequence of write and read pulses illuminates the source ensemble. The single photon generation is heralded by probabilistic detection of a Raman scattered field 1 from a write pulse. Conditioned on the heralding signal, a strong read pulse maps the excitation into a photonic mode, field 2, with probability of $50 \%$, which then propagates to the setup described in Fig. 1]. The resulting conditional probability to have a single photon, field 2 , at the face of memory ensemble is $15 \%$. The heralding signal triggers a control logic which disables the single-photon source and all associated laser beams for the programmable duration of the storage process for the quantum interface. 


\section{EIT storage and retrieval.}

The coherent interface between the signal modes and collective spin waves is achieved by dynamically controlling the EIT window $\Omega_{c}(t)$, defined by the atomlight interaction of a resonant control field. A quantum field propagating through an externally controlled dressed state medium is best described as a slow-light, dark-state polariton (DSP), $\hat{\Psi}(z, t)$ 26], a coherent mixture of matter-like and photonic excitations, expressed as

$$
\hat{\Psi}(z, t)=\cos \theta(t) \hat{\varepsilon}_{\text {signal }}-\sin \theta(t) \sqrt{N} \hat{\sigma}_{a b}
$$

where $\cos ^{2} \theta(t)=\frac{\Omega_{c}^{2}(t)}{\Omega_{c}^{2}(t)+g^{2} N}=\frac{v_{g}(t)}{c}, N$ is the number of atoms, $\hat{\sigma}_{a b}$ is the atomic coherence operator for ground states $|a\rangle,|b\rangle$, and $\Omega_{c}(t)$ is the Rabi frequency of control field. As the signal field propagates through the medium, the group velocity $v_{g}$ of the DSP is adiabatically reduced to zero as $\Omega_{c}(t)$ decreases to zero, thereby rotating $\theta(t)$ from a purely photonic state to a matter-like collective spin coherence. When the control field is re-activated, the collective spin excitation is coherently converted into a photonic mode in a time-reversal fashion.

* These authors contributed equally to this work.

$\ddagger$ To whom correspondence should be addressed. E-mail : hjkimble@caltech.edu

[1] Zoller, P. et al. Quantum information processing and communication, Strategic report on current status, visions and goals for research in Europe. Eur. Phys. J. D 36, 203-228 (2005).

[2] Clauser, J. F. \& Shimony, A. Bell's theorem. Experimental tests and implications. Rep. Prog. Phys. 41, 1881-1927 (1978).

[3] Briegel, H.-J., van Enk, S., Cirac, J. I. \& Zoller, P. in The Physics of Quantum Information, Bouwmeester, D., Ekert, A. \& Zeilinger, A. Eds. (Springer, Berlin, 2000).

[4] Duan, L.-M., Lukin, M., Cirac, J. I. \& Zoller, P. Longdistance quantum communication with atomic ensembles and linear optics. Nature 414, 413-418 (2001).

[5] Chou, C.-W. et al. Measurement-induced entanglement for excitation stored in remote atomic ensembles. Nature 438, 828-832 (2005).

[6] Chou, C.-W. et al. Functional quantum nodes for entanglement distribution over scalable quantum networks. Science 316, 1316-1320 (2007); published online 5 April 2007 (DOI 10.1126/science.1140300).

[7] Tan, S. M., Walls, D. F. \& Collett, M. J. Nonlocality of a single photon. Phys. Rev. Lett., 66, 252-255 (1991).

[8] Hessmo, B., Usachev, P., Hoshang, H. \& Gunner, B. Experimental demonstration of single photon nonlocality. Phys. Rev. Lett. 92, 180401 (2004).
[9] Jacques, V. et al. Experimental realization of wheeler's delayed-choice gedanken experiment. Science 315, 966968 (2007).

[10] Sangouard, N. et al. Long-distance entanglement distribution with single-photon sources. Phys. Rev. A 76, 050301(R) (2007).

[11] Lounis, B. \& Orrit, M. Single-photon sources. Rep. Prog. Phys. 68, 1129-1179 (2005).

[12] Dantan, A., Bramati, A., Pinerd, M. \& Giacobino, E. Manipulation and storage of optical field and atomic ensemble quantum states. Laser Phys. 15, 170-175 (2005)

[13] Quantum Information with Continuous Variables, Cerf, N. J., Leuchs, G. \& Polzik, E. S. Eds. (World Scientific Publishing, New Jersey, 2007).

[14] Sherson, J. F. et al. Quantum teleportation between light and matter. Nature 443, 557-560 (2006).

[15] Laurat, J. et al. Efficient retrieval of a single excitation stored in an atomic ensemble. Opt. Express 14, 6912-6918 (2006).

[16] Felinto, D. et al. Conditional control of the quantum states of remote atomic memories for quantum networking. Nature Physics 2, 844-848 (2006).

[17] Thompson, J. K., Simon, J., Loh, H. \& Vuletić, V. A high-brightness source of narrowband, identical-photon pairs. Science 313, 74-77 (2006).

[18] Matsukevich, D. N. et al. Deterministic single photons via conditional quantum evolution. Phys. Rev. Lett. 97, 013601 (2006).

[19] Chen, S. et al. Deterministic and storable single-photon source based on a quantum memory. Phys. Rev. Lett. 97, 173004 (2006).

[20] Laurat, J., Choi, K. S., Deng, H., Chou, C.-W. \& Kimble, H. J. Heralded entanglement between atomic ensembles: Preparation, decoherence, and scaling. Accepted in Phys. Rev. Lett., 99, 180504 (2007).

[21] Laurat, J. et al. Towards experimental entanglement connection with atomic ensembles in the single excitation regime. New J. Phys. 9, 207-220 (2007).

[22] Chen, Y.-A. et al. Memory-built-in quantum teleportation with photonic and atomic qubits. Preprint arXiv:0705.1256 (2007).

[23] Harris, S. E. Electromagnetically induced transparency. Phys. Today 50, 36-40 (1997).

[24] Hau, L. V., Harris, S. E., Dutton, Z. \& Behroozi, C. H. Light speed reduction to 17 metres per second in an ultracold atomic gas. Nature 397, 594-598 (1999).

[25] Kash, M. M. et al. Ultraslow group velocity and enhanced nonlinear optical effects in a coherently driven hot atomic gas. Phys. Rev. Lett. 82, 5229-5232 (1999).

[26] Fleischhauer, M. \& Lukin, M. D. Dark-state polaritons in electromagnetically induced transparency. Phys. Rev. Lett., 84, 5094-5097 (2000).

[27] Eisaman, M. D. et al. Electromagnetically induced transparency with tunable single-photon pulses. Nature $\mathbf{4 3 8}$, 837-841 (2005).

[28] Chaneliére, T. et al. Storage and retrieval of single photons transmitted between remote quantum memories. Nature 438, 833-836 (2005).

[29] Simon, J., Tanji, H., Ghosh, S. \& Vuletić, V. Singlephoton bus connecting spin-wave quantum memories. Nature Physics advanced online publication, 23 September 2007 (DOI 10.1038/nphys726).

[30] Novikova, I. et al. Optimal control of light pulse storage and retrieval. Phys. Rev. Lett. 98, 243602 (2007). 
[31] Wooters, W. K. Entanglement of formation of an arbitrary state of two qubits. Phys. Rev. Lett. 80, 2245-2248 (1998).

[32] de Riedmatten, H. et al. Direct measurement of decoherence for entanglement between a photon and stored atomic excitation. Phys. Rev. Lett. 97113603 (2006).

[33] Ringot, J., Szriftgiser, P. \& Garreau, J. C. Subrecoil raman spectroscopy of cold cesium atoms. Phys. Rev. A 65, 013403 (2001).

\section{Acknowledgement}

We gratefully acknowledge our ongoing collaboration with S. J. van Enk. This research is supported by the Disruptive Technologies Office and by the National Science Foundation. H.D. acknowledges support as Fellow of the Center for the Physics of Information at Caltech. J.L. acknowledges financial support from the European Union (Marie Curie Fellowship). 